\title{
Corrigendum: Catalytic subsurface etching of nanoscale channels in graphite
}

Maya Lukas, Velimir Meded, Aravind Vijayaraghavan, Li Song, Pulickel M. Ajayan, Karin Fink, Wolfgang Wenzel \& Ralph Krupke

Nature Communications 4:1379 doi: 10.1038/ncomms2399 (2013); Published 22 Jan 2013; Updated 23 Apr 2013

This Article contains errors in Fig. 3 and its legend. In Figs $3 b$ and d, the y axes are mislabelled and should have had units of pm. The scale bar lengths and STM parameters in the legend are also associated with the wrong panels, and should have referred to panels a and c. The correct version of Fig. 3 and its legend appears below.
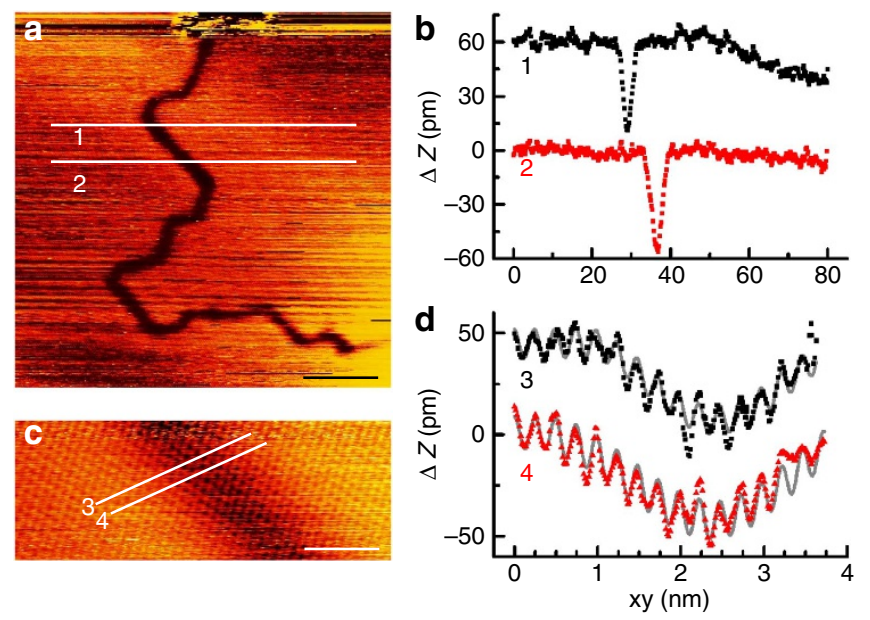

Figure 3 | High-resolution STM images and height profiles of a tunnel.

The height profiles (b) of the traces marked 1, 2 in the STM image (a) show a smooth submonolayer depression without edge enhancement. The atomically resolved STM image of the same area (c) shows an intact top graphene sheet. The height profiles (d) along the traces 3, 4 in $\mathbf{c}$ show unperturbed periodicity when fitted with a sine function. Black and red symbols: measured height from STM, grey line: sine function superimposed onto third order polynomial (flexed beam model). Scale bars, (a) $20 \mathrm{~nm}$, (c) $2 \mathrm{~nm}$, all images constant current mode, $U=0.7 \mathrm{~V}, I=0.98 \mathrm{nA}(\mathbf{a})$, $I=0.74 \mathrm{nA}(\mathbf{c})$. 